\title{
Extracorporeal life support in cardiogenic shock: Impact of acute versus chronic etiology on outcome
}

\author{
Vincenzo Tarzia, MD, ${ }^{\mathrm{a}}$ Giacomo Bortolussi, MD, ${ }^{\mathrm{a}}$ Roberto Bianco, MD, ${ }^{\mathrm{a}}$ Edward Buratto, MBBS, \\ Jonida Bejko, MD, ${ }^{\mathrm{a}}$ Massimiliano Carrozzini, MD, ${ }^{\mathrm{a}}$ Marco De Franceschi, BSS, ${ }^{\mathrm{a}}$ Dario Gregori, MA, PhD, ${ }^{\mathrm{b}}$ \\ Dario Fichera, CCP, MS, ${ }^{\mathrm{a}}$ Fabio Zanella, CCP, ${ }^{\mathrm{a}}$ Tomaso Bottio, MD, PhD, ${ }^{\mathrm{a}}$ and Gino Gerosa, $\mathrm{MD}^{\mathrm{a}}$
}

\begin{abstract}
Background: The role of extracorporeal life support (ECLS) in primary cardiogenic shock (PCS) is well established. In this study, we evaluated the impact of etiology on outcomes.

Methods: Between January 2009 and March 2013, we implanted a total of 249 patients with ECLS; we focused on 64 patients for whom peripheral ECLS was the treatment for PCS. Of these, 37 cases (58\%) were "acute" (mostly acute myocardial infarction: 39\%); $27(42 \%)$ had an exacerbation of "chronic" heart failure (dilated cardiomyopathy: 30\%; post-ischemic cardiomyopathy: $9 \%$; and congenital: $3 \%$ ).

Results: In the group with chronic etiology, 23 patients were bridged to a left ventricular assist device $(52 \%)$ or heart transplantation $(33 \%)$. In the group with acute etiology, ECLS was used as a bridge-to-transplantation in 3 patients $(8 \%)$, a bridge-to-bridge in $9(24 \%)$, and a bridge-to-recovery in $18(49 \%)$. One patient in each group was bridged to conventional surgery. Recovery of cardiac function was achieved in only the group with acute primary cardiogenic shock (18 vs 0 patients, $P=.0001$ ). A mean flow during support of $\leq 60 \%$ of the theoretic flow (body surface area $\times 2.4$ ) was a predictor of successful weaning $(P=.02)$. Median duration of ECLS support was 7 days (range: $2-11.5$ days). Nine patients (14\%) died during support; 30-day overall survival was 80\% (51 of 64 patients); and $59 \%$ of patients were discharged, in whom survival at 48 months was $90 \%$. Thirty-day survival was correlated with duration of ECLS support.
\end{abstract}

Conclusions: In "chronic" heart failure, ECLS represents a bridge to a ventricular assist device or heart transplantation, whereas in "acute" settings, it offers a considerable chance of recovery, and is often the only required therapy. (J Thorac Cardiovasc Surg 2015;150:333-40)

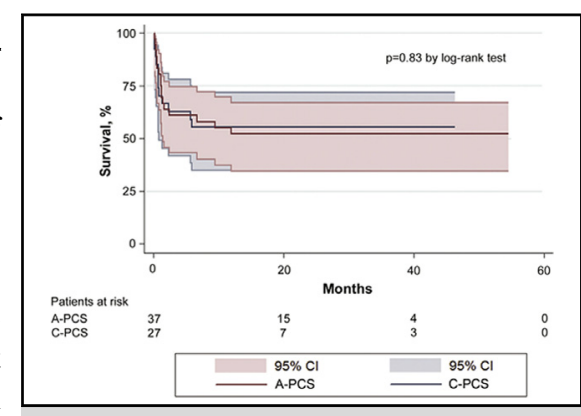

Overall survival of patients with primary cardiogenic shock with acute or chronic etiology.

Central Message

ECLS in C-PCS represents a bridge to VAD or heart-transplantation, whereas in A-PCS it offers chance of recovery. Better outcomes are observed with shorter and partial support.

Perspective

ECLS is a bridge-to-life and should be considered a first line treatment in PCS. Therapeutic strategy should be tailored considering the different outcomes with respect to etiology. Moreover, maintaining flows below $60 \%$ of the theoretical requirement and minimizing the duration of support appears to be the strategy which offers the best chance of survival.

See Editorial Commentary page 341.
Acute cardiogenic shock is a condition that continues to have very high mortality despite advances in medical therapy. ${ }^{1-3}$ Conventional treatment typically comprises inotrope infusions, vasopressors, and an intra-aortic balloon pump (IABP). ${ }^{1,4}$ When circulatory instability is refractory to these treatments, mechanical circulatory support represents

\footnotetext{
From the Division of Cardiac Surgery, ${ }^{\mathrm{a}}$ and Unit of Biostatistics, Epidemiology and Public Health, ${ }^{\mathrm{b}}$ Department of Cardiac, Thoracic and Vascular Sciences, University of Padua, Padua, Italy.

Received for publication April 18, 2014; revisions received Feb 9, 2015; accepted for publication Feb 20, 2015; available ahead of print April 11, 2015.

Address for reprints: Vincenzo Tarzia, MD, Division of Cardiac Surgery, Department of Cardiac, Thoracic and Vascular Sciences, University of Padua, Via Giustiniani 2, 35128 Padova, Italy (E-mail: v.tarzia@gmail.com).

$0022-5223 / \$ 36.00$

Copyright (c) 2015 by The American Association for Thoracic Surgery http://dx.doi.org/10.1016/j.jtcvs.2015.02.043
}

the only hope for survival, as indicated by current guidelines. 3

As most of these patients present with critical circulatory instability that requires urgent or emergent therapy, the chosen mechanical assistance should be able to be rapidly and easily implanted. For this reason, extracorporeal life support (ECLS) is the ideal "bridge to life," and increasingly, it is used to keep patients alive while the optimal therapeutic management is determined ("bridge to decision"). Management may follow 1 of 3 courses: (1) a "bridge to recovery," which involves patient recovery and weaning from ECLS; (2) a "bridge to transplant," which involves direct orthotopic heart transplantation; and (3) a "bridge to bridge," which involves placement of a ventricular assist device or total, artificial, longer-term support. ${ }^{5,6}$ Several large reports have been made on use of ECLS as a 


\section{Abbreviations and Acronyms \\ AMI = acute myocardial infarction \\ A-PCS $=$ acute primary cardiogenic shock \\ $\mathrm{C}-\mathrm{PCS}=$ chronic primary cardiogenic shock \\ ECLS = extracorporeal life support \\ $\mathrm{IABP}=$ intra-aortic balloon pump}

mechanical support in postcardiotomy patients ${ }^{3,7-9}$; but relatively few, most of which are small case-series studies, have focused on the role of ECLS in primary, cardiogenic shock (PCS) outside of the postcardiotomy setting. ${ }^{10-13}$

We present the results of our center's experience in the treatment of PCS with ECLS as a bridge to decision. Furthermore, we evaluated the impact of etiology on patient outcomes by comparing refractory PCS secondary to acute myocardial infarction (AMI), myocarditis, pulmonary embolism, and postpartum cardiomyopathy with acute decompensation of a chronic cardiomyopathy, including dilated cardiomyopathy, ischemic cardiomyopathy, and adult congenital heart diseases. In addition, we analyzed whether duration and magnitude of support predict weaning and survival.

\section{METHODS \\ Patients}

Between January 2009 and March 2013, a total of 249 patients were treated with ECLS in our center. We prospectively analyzed 64 patients implanted with the PLS (MAQUET, Cardiopulmonary AG, Hirrlingen, Germany) ECLS system for treatment of cardiogenic shock refractory to maximal inotropic and vasopressor therapy and treatment with IABP therapy, excluding postcardiotomy, pediatric, and respiratory supports. We divided patients into 2 groups, according to etiology. A total of 37 were assigned to the group with acute primary cardiogenic shock (A-PCS), for whom the primary cause was an acute event in a previously healthy heart, including 26 cases of AMI, 4 of myocarditis, 6 of pulmonary embolism, and 1 of postpartum cardiomyopathy. In the second group were 27 patients with chronic primary cardiogenic shock (C-PCS), in whom the etiology was an acute deterioration of a chronic cardiomyopathy, including 20 who had dilated cardiomyopathy, 5 who had ischemic cardiomyopathy (C-PCS), and 2 who had grownup congenital heart diseases (Table 1). Written informed consent was obtained from all patients, and the study was approved by our institutional review board.

\section{Criteria for Extracorporeal Life Support Installation}

Patients who met the criteria of profound cardiogenic shock due to pump failure were candidates for ECLS. Profound shock was defined as: systolic blood pressure of $<75 \mathrm{~mm} \mathrm{Hg}$; a cardiac index of $\leq 1.8 \mathrm{~L} /$ minute $/ \mathrm{m}^{2}$, with left ventricular end-diastolic pressure of $>20 \mathrm{~mm} \mathrm{Hg}$, despite multiple, high-dose, intravenous inotropic agents (dopamine $\geq 10 \mu \mathrm{g} / \mathrm{kg} /$ minute, dobutamine $\geq 10 \mu \mathrm{g} / \mathrm{kg} /$ minute, epinephrine $\geq 0.1 \mu \mathrm{g} / \mathrm{kg} /$ minute, and norepinephrine $\geq 0.1 \mu \mathrm{g} / \mathrm{kg} /$ minute) and/or IABP treatment, in association with clinical signs of pulmonary congestion; and impaired end-organ perfusion (renal, respiratory, and hepatic failure and altered mental status). ${ }^{14}$ The diagnosis of impaired end-organ function required $\geq 1$ of the following: altered mental status; cold, clammy skin and extremities; oliguria with urine output of $<30 \mathrm{ml}$ per hour; respiratory failure, defined as partial pressure of oxygen in arterial blood $<60 \mathrm{~mm} \mathrm{Hg}$ and/or partial pressure of carbon dioxide in arterial blood $>45 \mathrm{~mm} \mathrm{Hg}$ (type I-II), or need for mechanical ventilation in the setting of cardiogenic shock (type IV); serum lactate level $>2.0 \mathrm{mmol}$ per liter; bilirubin or transaminases $>3$ times the upper normal limit set by the local laboratory; and multiorgan failure, defined as failure of $\geq 2$ organs, in addition to cardiac dysfunction., ${ }^{4,15}$ The only absolute contraindication to ECLS was presence of severe neurologic involvement after arrest (significant anisocoria and signs of decerebration or focality); age $>75$ years and severe peripheral vascular disease were considered relative contraindications. ${ }^{6}$

\section{Extracorporeal Life Support System}

The system used in our institute was the PLS. ${ }^{7,16}$ This system has a portable, "all-in-one" design, including an oxygenator (Quadrox D), a centrifugal pump (Rotaflow), and heparin-coated tubes, as well as an optional heat exchanger, with specific features to minimize thrombotic risk. ${ }^{7} 16$ Furthermore, it has obtained CE (European conformity) approval for 14 days of support.

\section{Extracorporeal Life Support Placement}

In our institute, when feasible, we opted to implant ECLS with the patient awake and breathing independently, with local anesthetic, at the bedside. Cannulation was performed using a percutaneous veno-arterial Seldinger technique, with a venous drainage cannula ( $18 \mathrm{~F}$ to $28 \mathrm{~F}$ ) placed in the femoral vein, and an arterial return cannula (18F to $22 \mathrm{~F}$ ) placed in the femoral artery. When this strategy was unsuccessful or contraindicated (eg, by significant peripheral vascular disease, with small femoral vessels), the patient was taken to the operating theater, sedated, and intubated.

At the surgeon's discretion, 1 of the following cannulation techniques was chosen: subclavian or inguinal dissection and anastomosis of a 6- to 10-mm Dacron vascular graft (FlowWeave, Jotec GmbH, Hechingen, Germany) onto the subclavian or femoral artery for cannulation. In a single case, a sternotomy and central cannulation (a venous cannula in the right atrium and an arterial cannula in the ascending aorta) were used. Limb ischemia subsequent to percutaneous femoral arterial cannulation was handled via distal cannulation using a smaller cannula (either percutaneous or surgical), or by shifting the cannulation site.

\section{Anticoagulant Management}

Before placement of cannulae, a heparin bolus of $70 \mathrm{U} / \mathrm{Kg}$ (usually 5000 units) was administered to obtain an activated clotting time of 180 seconds. After this step, we performed activated partial thromboplastin time, international normalized ratio, and antithrombin assays 4 times per day; platelet counts, fibrinogen, and d-dimer assays were conducted once daily. Patients were kept anticoagulated with heparin maintenance, and with activated partial thromboplastin time in the range of 50 to 60 seconds. When d-dimer was elevated, or in cases of hemorrhagic or thrombotic complications, a thromboelastometry was carried out, and targeted therapy, based on its results, was initiated.

\section{Management of Extracorporeal Life Support}

Extracorporeal life support is intended to be a bridge-to-life, and at first, all patients need full-flow support (patient's estimated required cardiac output, calculated as: body surface area $\times 2.41 /$ minute). In this phase, stabilization is obtained, with cardiac function totally replaced by ECLS, which guarantees circulatory support and organ perfusion.

After this phase, some pulsatility may be observed, and this is a sign of initial recovery of cardiac function. In this second phase, we maintain inotropic support and the IABP when it is already present before ECLS implantation; we wake the patient to guarantee sympathetic tone; we perform weaning from mechanical ventilation, achieving extubation as soon as possible, to reduce pulmonary resistance; we start to reduce support, monitoring pulsatility and organ perfusion (lactate, urine output, central venous pressure, and pulmonary capillary wedge pressure). We maintain inotrope 
TABLE 1. Etiologies leading to ECLS implantation, in the overall population, the group who had A-PCS, and the group who had C-PCS

\begin{tabular}{lccc}
\hline \multicolumn{1}{c}{ Etiology } & $\begin{array}{c}\text { Overall } \\
(\mathbf{n = 6 4 )}\end{array}$ & $\begin{array}{c}\text { A-PCS } \\
(\mathbf{n = 3 7 )}\end{array}$ & $\begin{array}{c}\text { C-PCS } \\
(\mathbf{n = 2 7})\end{array}$ \\
\hline AMI & $26(41)$ & $26(70)$ & 0 \\
Myocarditis & $4(6)$ & $4(11)$ & 0 \\
Pulmonary embolism & $6(9)$ & $6(16)$ & 0 \\
Postpartum CM & $1(2)$ & $1(3)$ & 0 \\
Dilated CM & $20(31)$ & 0 & $20(74)$ \\
Ischemic CM & $5(8)$ & 0 & $5(19)$ \\
Congenital & $2(3)$ & 0 & $2(7)$ \\
\hline
\end{tabular}

Values are n (\%). A-PCS, Acute primary cardiogenic shock; $C$ - $P C S$, chronic primary cardiogenic shock; $A M I$, acute myocardial infarction; $C M$, cardiomyopathy.

therapy, to help the native heart open the aortic valve and empty the left ventricle, thus eliminating the need for a vent. If the patient remains stable, the third phase is initiated, which is a weaning protocol.

\section{Weaning Trial}

After obtaining hemodynamic stabilization and improvement of organ function (either neurologic, respiratory, renal, or hepatic), ECLS support was progressively decreased to $1 \mathrm{~L}$ per minute. Standard management involved serial echocardiograms during this phase, but we relied mostly on persistence of satisfactory hemodynamics and organ function, on low-to-medium dose inotropic support, to assess the feasibility of weaning from ECLS. Specifically, we performed echocardiographic evaluation of ventricular function (left ventricular ejection fraction $>35 \%$; good right ventricular contractility) and volume (absence of excessive ventricular distension, or severe tricuspid regurgitation), as well as clinical parameters, such as normal systemic pressure (systolic blood pressure $>85 \mathrm{~mm} \mathrm{Hg}$ ) and central venous pressure, normal blood lactate level, and urine output.

\section{Data Analysis}

Continuous variables are expressed as mean $\pm \mathrm{SD}$, or median (range). Categoric variables are summarized by reporting absolute frequency distribution and percentage; they were compared using $\chi^{2}$ analysis or the Fisher exact test, as appropriate. The Student $t$ test (for unpaired data) or the Mann-Whitney $U$ test was used to compare continuous variables, as appropriate (normal distribution was assessed by the Shapiro-Wilk normality test). Overall survival and survival after discharge were estimated by the Kaplan-Meier method.

To estimate effect of length of extracorporeal life support on 30-day survival, a multistate Cox Markov model ${ }^{17}$ was used, with the 3 states represented by death, recovery, and surgery procedure (ventricular assist device, heart transplantation, or conventional surgery). Thirty-day survival was computed using the cessation of extracorporeal life support as landmark point. ${ }^{18}$ Statistical analysis was performed using STATA software, release 10.0 (Stata Corporation, College Station, Tex), the R system, ${ }^{19}$ and the Time Dependent Cox libraries. ${ }^{20}$

\section{RESULTS}

Preoperative characteristics, mechanical support data, and outcomes are reported in Tables 2 and 3, respectively. Mean age was $50 \pm 16$ years, with no differences between the groups who had A-PCS versus C-PCS. The group who had A-PCS, versus C-PCS, had a higher incidence of: malignant arrhythmias (49\% vs $41 \%)$; cardiopulmonary resuscitation, either in the preceding 72 hours $(70 \%$ vs $22 \%)$ or during ECLS implantation $(38 \%$
TABLE 2. Preoperative characteristics and implantation technique

\begin{tabular}{|c|c|c|c|c|}
\hline $\begin{array}{l}\text { Characteristics } \\
\text { and techniques }\end{array}$ & $\begin{array}{r}\text { Overall } \\
(\mathrm{n}=64) \\
\end{array}$ & $\begin{array}{c}\text { A-PCS } \\
(\mathbf{n}=\mathbf{3 7}) \\
\end{array}$ & $\begin{array}{c}\text { C-PCS } \\
(\mathbf{n}=\mathbf{2 7}) \\
\end{array}$ & $\begin{array}{c}P \\
\text { value }\end{array}$ \\
\hline \multicolumn{5}{|l|}{ Preoperative characteristics } \\
\hline Age (y) & $50 \pm 16$ & $52 \pm 15$ & $48 \pm 17$ & .38 \\
\hline Gender, female & $12(19)$ & $10(27)$ & $2(7)$ & .004 \\
\hline $\operatorname{BSA}\left(\mathrm{m}^{2}\right)$ & $1.8 \pm 0.2$ & $1.9 \pm 0.2$ & $1.8 \pm 0.1$ & .001 \\
\hline Malignant arrythmia & $29(45)$ & $18(49)$ & $11(41)$ & .04 \\
\hline CPR within $72 \mathrm{~h}$ & $32(50)$ & $26(70)$ & $6(22)$ & $<.000$ \\
\hline No. of inotropes & $1.9 \pm 1$ & $1.7 \pm 1$ & $2.1 \pm 1.1$ & .009 \\
\hline IABP & $25(39)$ & $19(51)$ & $6(22)$ & .001 \\
\hline Respiratory failure & $55(86)$ & $32(86)$ & $23(85)$ & .04 \\
\hline Mechanical ventilation & $46(72)$ & $29(78)$ & $17(63)$ & .01 \\
\hline Renal failure & $32(50)$ & $14(38)$ & $18(67)$ & .001 \\
\hline $\mathrm{CVVH}$ & $8(13)$ & $4(11)$ & $4(15)$ & .71 \\
\hline Hepatic failure & $20(31)$ & $7(19)$ & $13(48)$ & .000 \\
\hline Multi-organ failure & $24(38)$ & $12(32)$ & $12(44)$ & .32 \\
\hline $\begin{array}{l}\text { APACHE IV score for } \\
\text { predicted mortality }(\%)\end{array}$ & $61 \pm 16$ & $58 \pm 16$ & $65 \pm 17$ & .38 \\
\hline \multicolumn{5}{|l|}{ Implantation technique } \\
\hline Bedside & $31(48)$ & $21(57)$ & $10(37)$ & .11 \\
\hline Salvage CPR & $17(27)$ & $14(38)$ & $3(11)$ & .02 \\
\hline Peripheral F-F & $51(80)$ & $32(86)$ & $19(70)$ & .11 \\
\hline Peripheral S-F & $12(19)$ & $4(11)$ & $8(30)$ & .1 \\
\hline Upgrade from F-F to S-F & $9(14)$ & $4(11)$ & $5(19)$ & .48 \\
\hline Central & $1(1)$ & $1(3)$ & 0 & .04 \\
\hline
\end{tabular}

Values are $\mathrm{n}(\%)$ or mean $\pm \mathrm{SD}$, unless otherwise indicated. $P$ values are for A-PCS versus C-PCS. A-PCS, Acute primary cardiogenic shock; $C$-PCS, chronic primary cardiogenic shock; $B S A$, body surface area; $C P R$, cardiopulmonary resuscitation; $I A B P$, intra-aortic balloon pump; $C V V H$, continuous veno-venous hemofiltration; APACHE, Acute Physiology and Chronic Health Evaluation; F-F, femoro-femoral; $S-F$, subclavian-femoral.

vs $11 \%)$; IABP treatment $(51 \%$ vs $22 \%)$; and mechanical ventilation ( $78 \%$ vs $63 \%)$.

In the group who had C-PCS, versus A-PCS, we observed a greater rate of end-organ dysfunction, demonstrated by the higher number of inotropes (2.1 vs 1.7), and renal $(67 \%$ vs $38 \%)$ and hepatic failure $(48 \%$ vs $19 \%)$. The Acute Physiology and Chronic Health Evaluation (APACHE) IV score indicated a $61 \% \pm 16 \%$ predicted mortality, with no differences between the 2 groups. Veno-arterial ECLS was implanted peripherally in all but 1 case. A total of 51 patients were treated with femorofemoral cannulation, and 12 with subclavian-femoral ECLS. Nine patients required shifting of the cannulation site from the femoral to the subclavian artery, because of limb ischemia or issues in femoral cannulation (Table 2).

The median duration of ECLS support was 7 days (interquartile range: 2-11.5 days), with no differences between the 2 groups. Mean percentage of theoretic flow was $61 \% \pm 15 \%$. Patients in the C-PCS group required greater support, compared with those who had A-PCS $(67 \%$ vs $57 \%)$, to achieve adequate organ perfusion, as confirmed by the absence of difference in serum lactate levels (expressed as the median of the peak lactate on a daily basis). As expected, given that the frequency of AMI was 
TABLE 3. Parameters, outcomes, and complications of ECLS support

\begin{tabular}{|c|c|c|c|c|}
\hline Parameter, outcome, complication & Overall $(n=64)$ & A-PCS $(n=37)$ & C-PCS $(n=27)$ & $P$ value \\
\hline \multicolumn{5}{|l|}{ Parameter } \\
\hline Duration (d) & $7(2-11.5)$ & $7(2.75-10.25)$ & $7(2-12.75)$ & .86 \\
\hline Flow (\% of theoretic CO) & $61 \pm 15$ & $57 \pm 13$ & $67 \pm 15$ & .004 \\
\hline No. of inotropes & $2.6 \pm 1.1$ & $2.2 \pm 1.1$ & $3.1 \pm 0.8$ & $<.001$ \\
\hline Serum lactates $(\mathrm{mmol} / \mathrm{L})$ & $2.55(1.7-3.6)$ & $2.3(1.55-3.25)$ & $2.85(1.9-4.3)$ & .14 \\
\hline TnI peak $(\mu \mathrm{g} / \mathrm{L})$ & $3.75(0.33-100)$ & $53(2.2-231.9)$ & $0.47(0.15-1.98)$ & .0007 \\
\hline \multicolumn{5}{|l|}{ Outcome } \\
\hline BTD & $9(14)$ & $6(16)$ & $3(11)$ & .72 \\
\hline BTR & $18(28)$ & $18(49)$ & 0 & $<.0001$ \\
\hline BTS & $2(3)$ & $1(3)$ & $1(4)$ & .04 \\
\hline BTB & $23(36)$ & $9(24)$ & $14(52)$ & .001 \\
\hline BTT & $12(19)$ & $3(8)$ & $9(33)$ & .001 \\
\hline Mortality in ECLS & $9(14)$ & $6(16)$ & $3(11)$ & .72 \\
\hline Mortality, 30-d & $13(20)$ & $6(16)$ & $7(26)$ & .02 \\
\hline Discharged from hospital & $37(58)$ & $22(59)$ & $15(56)$ & .75 \\
\hline \multicolumn{5}{|l|}{ Complications during ECLS } \\
\hline Neurologic & $12(19)$ & $8(22)$ & $4(15)$ & .49 \\
\hline Limb ischemia & $9(14)$ & $6(16)$ & $3(11)$ & .72 \\
\hline Leg amputation & 0 & 0 & 0 & 1.00 \\
\hline Bleeding & $13(20)$ & $8(22)$ & $5(19)$ & .76 \\
\hline Hemolysis & $1(2)$ & $1(3)$ & 0 & 1.00 \\
\hline Renal failure & $25(39)$ & $12(32)$ & $13(48)$ & .20 \\
\hline $\mathrm{CVVH}$ & $22(34)$ & $10(27)$ & $12(44)$ & .14 \\
\hline Oxygenator change & $14(22)$ & $5(14)$ & $9(33)$ & .06 \\
\hline Malfunction & $4(6)$ & $2(5)$ & $2(7)$ & 1.00 \\
\hline ARDS/pulmonary congestion & $5(8)$ & $1(3)$ & $4(15)$ & .15 \\
\hline Sepsis & $9(14)$ & $3(8)$ & $6(22)$ & .15 \\
\hline MOF, post & $14(22)$ & $8(22)$ & $6(22)$ & .95 \\
\hline
\end{tabular}

Values are $\mathrm{n}(\%)$, mean $\pm \mathrm{SD}$, or median (interquartile range), unless otherwise indicated. $P$ values are for A-PCS versus C-PCS. ECLS, Extracorporeal life support; $A$-PCS, acute primary cardiogenic shock; $C$-PCS, chronic primary cardiogenic shock; $C O$, cardiac output; $T n I$, troponin I; $B T D$, bridge-to-decision; $B T R$, bridge-to-recovery; $B T S$, bridge-toconventional surgery; $B T B$, bridge-to-bridge; $B T T$, bridge-to-transplant; $C V V H$, continuous veno-venous hemofiltration; $A R D S$, acute respiratory distress syndrome; $M O F$, multi-organ failure.

higher in the A-PCS group, peak troponin was significantly greater in this group.

The outcomes of ECLS support differed significantly between the 2 groups. In the group who had C-PCS, 23 patients were bridged to either a left ventricular assist device $(52 \%)$ or heart transplantation $(33 \%)$. Conversely, in the group who had A-PCS, ECLS was used as a bridge-to-transplantation in 3 patients $(8 \%)$, a bridge-tobridge in 9 patients (24\%), and a bridge-to-recovery in 18 patients $(49 \%)$. One patient in each group was bridged to conventional surgery.

Recovery of cardiac function was achieved only in the group who had A-PCS (18 vs 0 patients, $P=.0001$ ). The group with A-PCS, versus C-PCS, were comparable in: mortality during ECLS support (16\% in A-PCS vs $11 \%$ in C-PCS), hospital discharge (59\% in A-PCS vs $56 \%$ in C-PCS) and survival at 1-year follow-up (49\% in group A-PCS vs $44 \%$ in C-PCS), However, 30-day mortality was significantly greater in the group who had C-PCS, versus A-PCS $(26 \%$ vs $16 \%, P=.02)$. Complications during support were similar in the 2 groups (Table 3 ).
Evaluation of the impact of ECLS duration on survival indicated that risk of dying at 30 days increased at a constant rate throughout the duration of ECLS (for every 4 days on ECLS, the hazard ratio is $6.11,95 \%$ confidence interval: 1.15-32.3) in patients undergoing a surgical procedure. In patients who recovered, the risk of dying at 30 days increased steadily only after 9 days of ECLS (for every 2 days on ECLS, the hazard ratio is 14.53 , 95\% confidence interval: 1.12-189.0). The confidence intervals are huge because of the low sample size of patients on ECLS for $>16$ days (Figure 1). Analysis of flow rates has demonstrated that in the A-PCS group, the required percentage of support is a significant predictor of recovery (Figure $2, A[P=.03]$ ). In fact, we have shown that flow rates $<60 \%$ correlate with the probability of recovery $(P=.02)$. A subgroup of particular interest is patients who have AMI, in whom this phenomenon is particularly strong (Figure 2, $B[P=.01]$ ).

Overall survival did not differ between the 2 groups; for A-PCS and C-PCS, respectively, survival was $73 \%$ versus $70 \%$ at 1 month; $57 \%$ versus $56 \%$ at 6 months; and $51 \%$ 


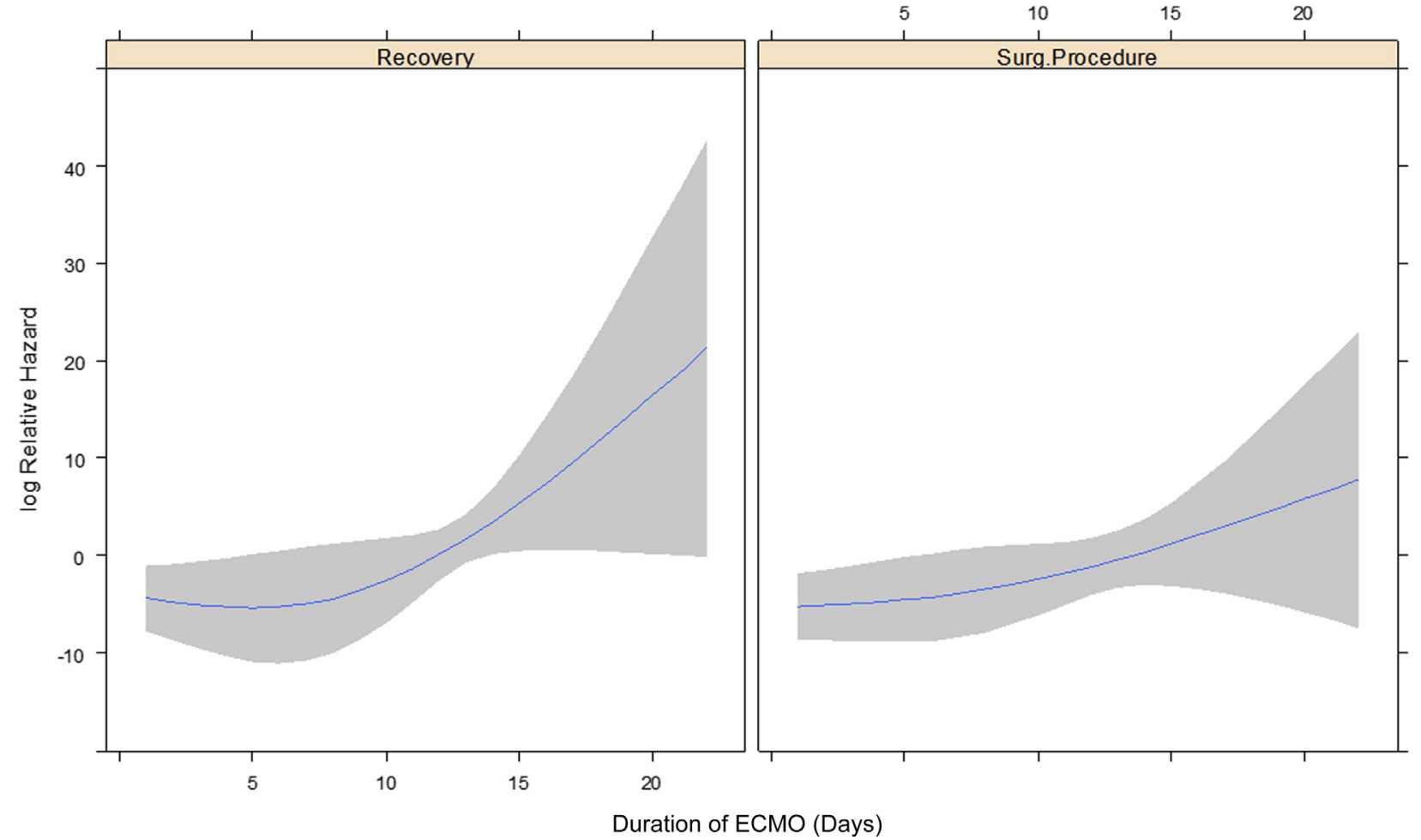

FIGURE 1. Impact of duration of ECLS on 30-day survival for the 2 distinct states: "recovery" and "surgical procedure." Risk of dying at 30 days increases at a constant rate throughout the duration of ECLS for patients in the surgical procedure stage. In patients who recovered, the risk of dying at 30 days increases steadily only after 9 days on ECLS. Surg, Surgery; ECMO, extracorporeal membrane oxygenation.

versus $55 \%$ at 1 year (Figure 3, A). The overall actuarial survival of the cohort after discharge was $89 \%$ at 1 year (Figure 3,B). A result of particular interest was the survival of those patients who recovered cardiac function, which was $83 \%$ at 1 -year follow up (Figure $3, C$ ).

\section{DISCUSSION}

The aim of this study was to evaluate the impact of etiology—in particular, acute versus chronic-on the outcome of patients in primary cardiogenic shock who are treated with ECLS. The outcomes assessed were death during ECLS support, 30-day mortality, hospital discharge, and survival at follow up, both overall and in the 2 etiology groups. The 2 groups were comparable in terms of their preimplantation condition and postimplantation complications.

Differences between the groups could be attributed to the differing etiology of cardiogenic shock. In the group with C-PCS, the shock represents the end stage of a progressive decline in cardiac function with chronic structural damage. This scenario was not seen in the group with A-PCS, in which the most common cause of cardiogenic shock was AMI. In the analysis of primary endpoints, the only difference between the groups was that 30-day mortality was higher with C-PCS, compared with A-PCS.
These data are consistent with the recent series published by Bermudez and colleagues, ${ }^{6}$ but differ regarding long-term survival. In our series, the 2 groups were comparable in mortality during support, hospital discharge, and even survival at follow up. Overall, the outcomes are in line with the literature, as is overall survival. ${ }^{13,21}$ In light of these results, a reasonable conclusion is that use of ECLS allows survival to be maximized in patients who are otherwise untreatable and face an extremely severe prognosis, independent of etiology of the shock, and of whether it is acute or chronic. In our experience, unlike that of Bermudez et al, ${ }^{6}$ patients in a chronic setting can additionally benefit from ECLS as a bridge to candidacy for a ventricular assist device or a transplant.

As secondary endpoints, we looked at the proportion of patients who had recovery of cardiac function (bridge-to-recovery), to determine if the duration and magnitude of support are predictors of recovery and survival. In keeping with the markedly different profiles of the 2 groups, recovery occurred in only the group with A-PCS. This difference was highly significant and reinforces the idea that cardiogenic shock, in the context of chronic progressive cardiac failure, represents the terminal stage of the disease, which cannot be remedied without replacing the heart's pumping function. As a result, in the group who had 

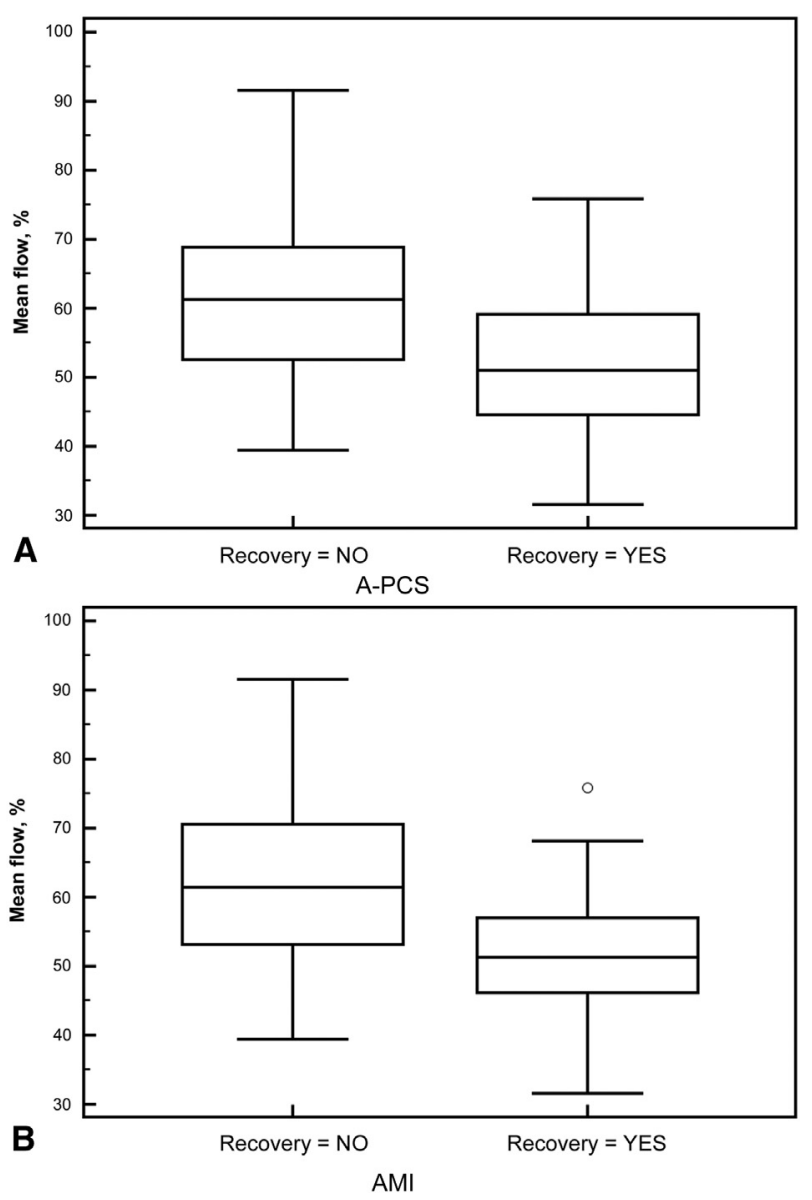

FIGURE 2. Analysis of mean flow versus (A) recovery in the group who had A-PCS $(P=.03)$; and $(\mathrm{B})$ recovery in the group who had AMI $(P=.01)$. In the box-and-whisker plot, the central box represents the values from the lower to upper quartile (25th-75th percentile). The middle line represents the median. The horizontal line extends from the minimum to the maximum value, excluding one outlier value, which is displayed as separate circle. A-PCS, Acute primary cardiogenic shock; AMI, acute myocardial infarction.

C-PCS, ECLS acted essentially either as a bridge to a ventricular assist device or as a bridge-to-transplant. Conversely, in the group who had A-PCS, ECLS support temporarily rests the damaged myocardium, allowing it to recover functionality. ${ }^{19}$ In patients with refractory shock secondary to an acute etiology, ECLS offers a substantial chance of recovery, often representing, after failed percutaneous coronary intervention, the only therapy required.

In patients with the best 30-day survival, the duration of ECLS was significantly shorter. Specifically, for patients recovering their cardiac function, risk of death does not increase until the 9th day; our data suggest that recovery beyond this limit, although possible, is burdened by higher mortality. Conversely, patients in need of additional surgical procedures, owing to the constant increase in
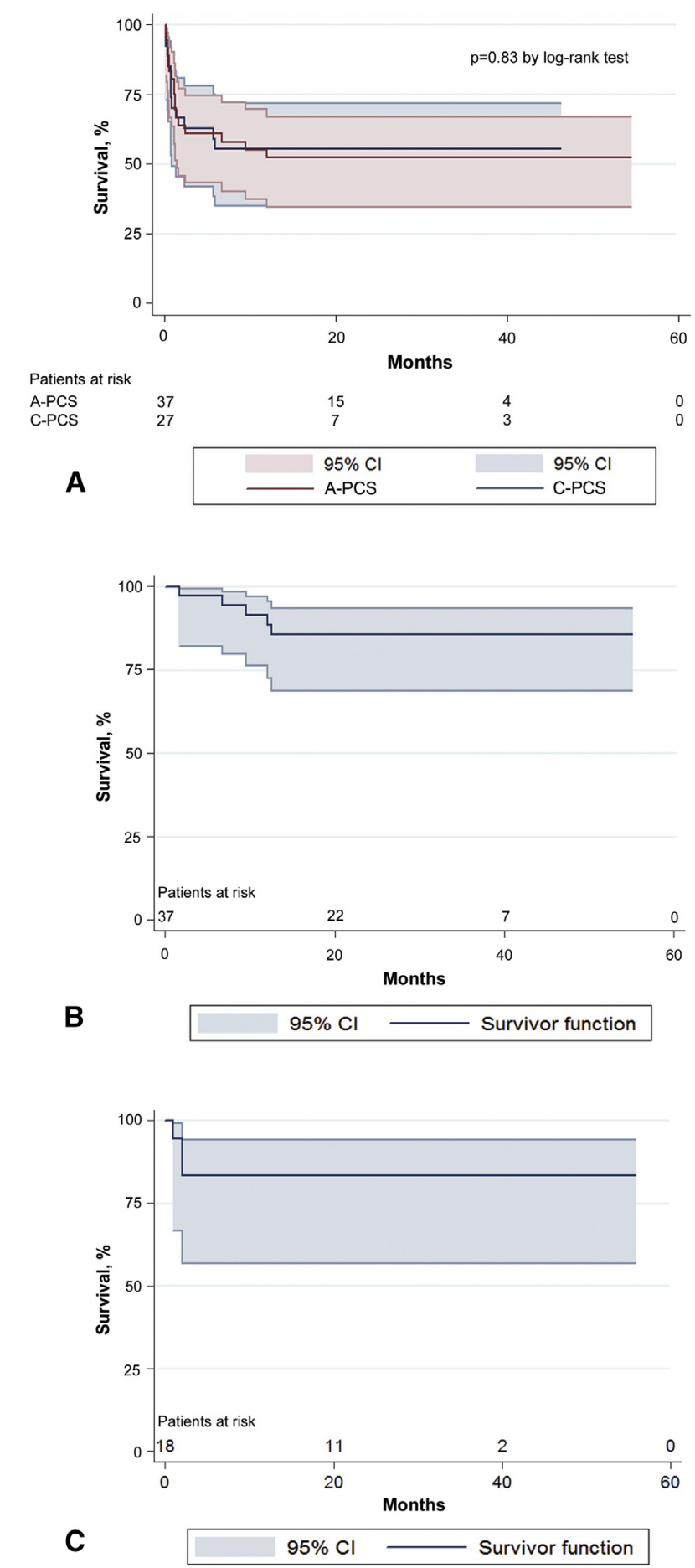

FIGURE 3. Kaplan-Meier estimates of actuarial survival in 3 populations: (A) all patients; (B) discharged patients; and (C) bridge-to-recovery subgroup after discharge. A-PCS, Acute primary cardiogenic shock; $C$-PCS, chronic primary cardiogenic shock; $C I$, confidence interval.

mortality over time, would likely benefit from early planning of a therapeutic strategy. Potentially, the shorter duration of support reflects, at least partly, a less substantial systemic compromise. 
Another part of this study is related to the management of the flow rates. After the first phase of "resuscitation" at full flow, we aimed to reduce the support, whenever possible. Specific strategies make this possible. First, the use of ECLS reduces venous return and hence the preload of the left ventricle, thus reducing the work it is required to do. Second, ECLS provides most of the splanchnic circulation, but does not increase the afterload on the left ventricle as much as full support would. Finally, with the help of inotropes, the native heart is often able to open the aortic valve and empty the left ventricle, thus eliminating the need for a vent and providing most of the coronary and cerebral perfusion.

Within the group with A-PCS, analyses of flow rates demonstrate that the maintenance of support at $<60 \%$ of the theoretic requirement was significantly associated with recovery of cardiac function, which likely reflects a less-severe initial myocardial condition and a greater degree of reversibility. Of particular interest in the group of patients who had A-PCS is the subgroup who had AMI, because of both the epidemiologic significance of this condition and its high frequency within this group $(n=26)$. In this subgroup, the proportion of patients who recover cardiac function is very promising, totaling $42 \%$.

These results are comparable to those in the literature, particularly in studies reporting outcomes for patients with cardiogenic shock secondary to AMI. ${ }^{21}$ A reasonable possibility is that, in this group, ECLS helps to contain the damage caused by ischemia-reperfusion injury and evolution of the infarcted area, by directly reducing the afterload against which the heart has to work, and hence reducing the oxygen demand of the myocardium. ${ }^{22}$ In this subgroup, the degree of ECLS support was a predictor of cardiac function recovery: Patients who experienced recovery had mean flows of $55 \%$ of the theoretic predicted requirement.

The analysis of all patients as a single cohort showed that 30-day survival was approximately $80 \%$, and discharge from the hospital was approximately $60 \%$. A particularly encouraging result was $89 \%$ survival at 2 -year follow up in patients discharged from the hospital. The results for the subgroup with shock due to AMI was consistent with these findings, but peculiar in that ECLS was the only necessary treatment after failed percutaneous coronary intervention, for $42 \%$ of the patients, and provided long-term survival on the order of $80 \%$. These results support the use of ECLS in PCS, whereas treatment with IABP was recently shown to confer no benefit in terms of mortality. ${ }^{23}$

\section{CONCLUSIONS}

Extracorporeal life support is intended as a bridge-to-life and should be considered as a first line of support in patients with refractory cardiogenic shock. In the setting of a chronic decline in cardiac function, ECLS represents a bridge to implantation of a ventricular assist device or to an orthotopic heart transplant. In patients with refractory shock due to acute etiologies, ECLS offers a substantial chance of recovery, often representing, after failed percutaneous coronary intervention, the only necessary treatment. In our experience, the magnitude and duration of the ECLS support significantly affects the chance of weaning, and of survival, reflecting a less severe initial myocardial condition and a greater degree of reversibility. Maintaining flows at approximately $60 \%$ of the theoretic requirement and minimizing the duration of support seems to be the strategy that offers the best chance of survival.

\section{Conflict of Interest Statement}

Authors have nothing to disclose with regard to commercial support.

\section{References}

1. Goldstein D, Neragi-Miandoab S. Mechanical bridge to decision: What are the options for the management of acute refractory cardiogenic shock? Curr Heart Fail Rep. 2011;8:51-8

2. Koerner MM, Jahanyar J. Assist devices for circulatory support in therapyrefractory acute heart failure. Curr Opin Cardiol. 2008;23:399-406.

3. Russo CF, Cannata A, Lanfranconi M, Bruschi G, Milazzo F, Paino R, et al. Veno-arterial extracorporeal membrane oxygenation using Levitronix centrifugal pump as bridge to decision for refractory cardiogenic shock. J Thorac Cardiovasc Surg. 2010;140:1416-21.

4. McMurray JJ, Adamopoulos S, Anker SD, Auricchio A, Böhm M, Dickstein K, et al. ESC Guidelines for the diagnosis and treatment of acute and chronic heart failure 2012. Eur Heart J. 2012;33:1787-847.

5. Marasco SF, Lukas G, McDonald M, McMillan J, Ihle B. Review of ECMO (extra corporeal membrane oxygenation) support in critically ill adult patients. Heart Lung Circ. 2008;17:S41-7.

6. Bermudez CA, Rocha RV, Toyoda Y, Zaldonis D, Sappington PL, Mulukutla S, et al. Extracorporeal membrane oxygenation for advanced refractory shock in acute and chronic cardiomyopathy. Ann Thorac Surg. 2011;92:2125-31.

7. Formica F, Avalli L, Martino A, Maggioni E, Muratore M, Ferro O, et al. Extracorporeal membrane oxygenation with a poly-methylpentene oxygenator (Quadrox D). The experience of a single Italian centre in adult patients with refractory cardiogenic shock. ASAIO J. 2008;54:89-94.

8. Rastan AJ, Dege A, Mohr M, Doll N, Falk V, Walther T, et al. Early and late outcomes of 517 consecutive adult patients treated with extracorporeal membrane oxygenation for refractory postcardiotomy cardiogenic shock. J Thorac Cardiovasc Surg. 2010;139:302-11.

9. Hei F, Lou S, Li J, Yu K, Liu J, Feng Z, et al. Five-year results of 121 consecutive patients treated with extracorporeal membrane oxygenation at Fu Wai hospital. Artif Organs. 2011;35:572-8.

10. Arlt M, Philipp A, Voelkel S, Camboni D, Rupprecht L, Graf BM, et al. Hand-held minimised extracorporeal membrane oxygenation: a new bridge to recovery in patients with out-of-centre cardiogenic shock. Eur J Cardiothorac Surg. 2011;40:689-94.

11. Tang GH, Malekan R, Kai M, Lansman SL, Spielvogel D. Peripheral venoarterial extracorporeal membrane oxygenation improves survival in myocardia infarction with cardiogenic shock. J Thorac Cardiovasc Surg. 2013;145:e32-3.

12. Moraca RJ, Wanamaker KM, Bailey SH, McGregor WE, Murali S, Benza R, et al. Salvage peripheral extracorporeal membrane oxygenation using Cobe Revolution centrifugal pump as a bridge to decision for acute refractory cardiogenic shock. J Card Surg. 2012;27:521-7.

13. Chen YS, Yu HY, Huang SC, Chiu KM, Lin TY, Lai LP, et al. Experience and result of extracorporeal membrane oxygenation in treating fulminant myocarditis with shock: What mechanical support should be considered first? J Heart Lung Transplant. 2005;24:81-7.

14. Tsao NW, Shih CM, Yeh JS, Kao YT, Hsieh MH, Ou KL, et al Extracorporeal membrane oxygenation-assisted primary percutaneous coronary intervention may improve survival of patients with acute myocardial infarction complicated by profound cardiogenic shock. J Crit Care. 2012;27:530.e1-11.

15. Extracorporeal Life Support Organization Guidelines for cardiopulmonary extracorporeal life support, version 1.3. Available at: www.elso.org/Resources/ Guidelines.aspx. Accessed March 1, 2014. 
16. Arlt M, Philipp A, Voelkel S, Rupprecht L, Mueller T, Hilker M, et al. Extracorporeal membrane oxygenation in severe trauma patients with bleeding shock. Resuscitation. 2010;81:804-9.

17. Meira-Machado L, Cadarso-Suarez C, De Una-Alvarez J. tdc.msm: an R library for the analysis of multi-state survival data. Comput Methods Programs Biomed. 2007:86:131-40.

18. Van Houwelingen HC. Dynamic prediction by landmarking in event history analysis. Scand J Stat. 2007;34:70-85.

19. R Foundation for Statistical Computing/ R Development Core Team. The R Project for Statistical Computing. Available at: http://www.R-project.org. Accessed March 1, 2014.

20. Meira-Machado L, De Una-Alvarez J, Cadarso-Suarez C, Andersen PK Multi-state models for the analysis of time-to-event data. Stat Methods Med Res. 2009;18:195-222.
21. Wu MY, Lee MY, Lin CC, Chang YS, Tsai FC, Lin PJ. Resuscitation of non-postcardiotomy cardiogenic shock or cardiac arrest with extracorporeal life support: the role of bridging to intervention. Resuscitation. 2012;83:976-81.

22. Schopka S, Philipp A, Lunz D, Camboni D, Zacher R, Rupprecht L, et al. Single-center experience with extracorporeal life support in 103 nonpostcardiotomy patients. Artif Organs. 2013;37:150-6.

23. Thiele H, Zeymer U, Neumann FJ, Ferenc M, Olbrich HG, Hausleiter J, et al. Intraaortic balloon support for myocardial infarction with cardiogenic shock N Engl J Med. 2012;4:1287-96.

Key Words: Extracorporeal membrane oxygenation, ECMO, circulatory temporary support, shock, myocardial infarction, cardiomyopathy

Readers who found these articles interesting may also like to read the following papers found in recent and future issues of our sister publications, Seminars in Thoracic and Cardiovascular Surgery and Operative Techniques in Thoracic and Cardiovascular Surgery!

\section{Cardiothoracic Transplantation and Mechanical Circulatory Support}

News and Views: O. Howard Frazier. Mechanical Circulatory Assist Device Development at the Texas Heart Institute. Semin Thorac Cardiovasc Surg. Spring 2014;26(1):4-13.

State of the Art: Steven Singh. The State of the Art in Corporeal Membrane Oxygenation. Semin Thorac Cardiovasc Surg. Expected publication August 2015.

Carmelo Milano. Heart Transplantation after Left Ventricular Assist Device. Oper Tech Thorac Cardiovasc Surg. Expected publication November 2014.

Mani Daneshmand. Bilateral Sequential Lung Transplantation-evolution to simplify. Oper Tech Thorac Cardiovasc Surg. Summer 2014;19(2):138-151.

Charles Hoopes. Ambulatory Extracorporeal Membrane Oxygenation. Oper Tech Thorac Cardiovasc Surg. Summer 2014;19(2): 129-137.

Pankaj Saxena. Techniques for lung procurement following donation after circulatory death. Oper Tech Thorac Cardiovasc Surg. Expected publication February 2015.

Ashok Babu. Techniques for venoarterial ECMO support and conversion to temporary left ventricular Assist Device. Oper Tech Thorac Cardiovasc Surg. Expected publication February 2015.

Simon Maltais. Non-sternotomy approaches to left ventricular assist device placement. Oper Tech Thorac Cardiovasc Surg. Expected publication February 2015.

Anelechi Anyanwu. Non-Sternotomy Approaches to Left Ventricular Assist Device Placement: Combined Left Subcostal-Right Mini-Thoracotomy Technique. Oper Tech Thorac Cardiovasc Surg. Expected publication February 2015. 